ESAIM: PROCEEDINGS, December 2012, Vol. 38, p. 348-360

F. Coquel, M. Gutnic, P. Helluy, F. Lagoutière, C. Rohde, N. Seguin, Editors

\title{
FIRST ORDER TWO-SCALE PARTICLE-IN-CELL NUMERICAL METHOD FOR THE VLASOV EQUATION
}

\author{
Emmanuel Frénod ${ }^{1,2}$, Michä̈l Gutnic ${ }^{2}$ And Sever A. Hirstoaga ${ }^{2}$
}

\begin{abstract}
The aim of this work is to build an accurate numerical method for the simulation of the long time evolution of the Vlasov solution $f^{\varepsilon}$ with an electric field $E^{\varepsilon}=E_{0}+\varepsilon E_{1}$ for small $\varepsilon$. To this purpose, we use the Two-Scale Convergence to determine a first order approximation $F+\varepsilon F_{1}$ of $f^{\varepsilon}$. Then, by means of particle approximations we build an algorithm which is intended for providing a numerical approximation of $F+\varepsilon F_{1}$.

Résumé. On cherche à construire une méthode numérique pour l'évolution en temps long de la solution $f^{\varepsilon}$ de l'équation de Vlasov avec un champ électrique $E^{\varepsilon}=E_{0}+\varepsilon E_{1}$ pour $\varepsilon$ petit. À cet effet, on utilise la théorie de la convergence à deux échelles pour obtenir une approximation d'ordre un $F+\varepsilon F_{1}$ de $f^{\varepsilon}$, puis une méthode particulaire pour construire l'algorithme d'approximation numérique de $F+\varepsilon F_{1}$.
\end{abstract}

\section{CONTEnts}

1. Introduction

2. On Two-Scale Convergence statements. Main Result 349

\begin{tabular}{lll}
\hline 2.1. & Zeroth order approximation & 351
\end{tabular}

$\begin{array}{lll}2.2 . & \text { First order approximation } & 352\end{array}$

\begin{tabular}{lll}
\hline 3. & Numerical algorithm & 357 \\
\hline
\end{tabular}

\begin{tabular}{ll}
\hline References & 360
\end{tabular}

\section{INTRODUCTION}

In this paper, we are interested in the solution $f^{\varepsilon}$ to the following Vlasov problem set in a bi-dimensional phase space

$$
\left\{\begin{array}{l}
\frac{\partial f^{\varepsilon}}{\partial t}+\frac{v}{\varepsilon} \frac{\partial f^{\varepsilon}}{\partial r}+\left(E^{\varepsilon}-\frac{r}{\varepsilon}\right) \frac{\partial f^{\varepsilon}}{\partial v}=0 \\
f^{\varepsilon}(t=0, r, v)=f_{0}(r, v)
\end{array}\right.
$$

1 Université Européenne de Bretagne, LMBA (UMR CNRS 6205), Université de Bretagne-Sud, Centre Yves Coppens, Campus de Tohannic, F-56017, Vannes

${ }^{2}$ Inria Nancy-Grand Est, CALVI Project \& IRMA (UMR 7501), Université de Strasbourg, 7 rue René Descartes, F-67084 Strasbourg Cedex, France

(C) EDP Sciences, SMAI 2012 
where $f^{\varepsilon}:(t, r, v) \mapsto f^{\varepsilon}(t, r, v)$, for $t \in[0, T), r \in \mathbb{R}^{+}$and $v \in \mathbb{R}$. In 1.1), let

$$
E^{\varepsilon}(t, r)=E_{0}\left(t, \frac{t}{\varepsilon}, r\right)+\varepsilon E_{1}\left(t, \frac{t}{\varepsilon}, r\right)
$$

where $E_{i=0,1}:(t, \tau, r) \mapsto E_{i=0,1}(t, \tau, r)$ are regular and $2 \pi$-periodic with respect to $\tau$. Our purpose is to build a numerical method which is efficient for simulating the long time evolution of $f^{\varepsilon}$ in the limit $\varepsilon \rightarrow 0$

In Section 2, we use the Two-Scale Convergence to determine a first order approximation of $f^{\varepsilon}$, namely $f^{\varepsilon}(t, r, v) \approx F(t, t / \varepsilon, r, v)+\varepsilon F_{1}(t, t / \varepsilon, r, v)$ where $F:(t, \tau, r, v) \mapsto F(t, \tau, r, v)$ and $F_{1}:(t, \tau, r, v) \mapsto F(t, \tau, r, v)$ are $2 \pi$-periodic functions with respect to $\tau \in \mathbb{R}$.

We first recall in Section 2.1 that, for the zeroth order term, there exists a function $G:(t, q, u) \mapsto$ $G(t, q, u)$ solution of an initial boundary condition partial differential problem such that $F(t, \tau, r, v)=$ $G\left(t, \mathcal{R}^{\tau}(r, v)\right)$, where $\mathcal{R}^{\tau}$ is the 2D-rotation of angle $\tau$. In Section 2.2, we handle the first order term and show that there exist two functions $G_{1}:(t, q, u) \mapsto G_{1}(t, q, u)$ and $W:(t, \tau, q, u) \mapsto W(t, \tau, q, u)$ such that $F_{1}(t, \tau, r, v)=G_{1}\left(t, \mathcal{R}^{\tau}(r, v)\right)+W\left(t, \tau, \mathcal{R}^{\tau}(r, v)\right)$, where $W$ can be computed from $\nabla_{q, u} G$ and $G_{1}$ is the solution of an initial boundary condition partial differential problem.

In Section 3, we introduce a Particle-in-Cell based approximation to build a numerical algorithm which will allow us to solve numerically the problems satisfied by $G$ and $G_{1}$, and also to compute a numerical approximation of $W$.

\section{On Two-Scale Convergence statements. Main Result}

The concept of Two-Scale Convergence was introduced at the end of the 80's by Nguetseng [5,6] In 1992, Allaire gave a very understandable proof of this result 11. Then several authors used this theory to build numerical methods called Two-Scale Numerical Methods (see, for example, Frénod and Sonnendrücker [3, Frénod, Salvarani and Sonnendrücker [2], Mouton [4], and the references therein). We only recall here the definition of the Two-Scale Convergence.

Definition 2.1. A function $f^{\varepsilon}:(t, r, v) \in[0, T) \times \mathbb{R}^{2} \mapsto f^{\varepsilon}(t, r, v)$ Two-Scale converges as $\varepsilon \rightarrow 0$ to a function $F:(t, \tau, r, v) \in[0, T) \times \mathbb{R} \times \mathbb{R}^{2} \mapsto F(t, \tau, r, v) 2 \pi$-periodic in $\tau$, if:

$$
\int_{0}^{T} \int_{\mathbb{R}^{2}} f^{\varepsilon}(t, r, v)[\phi]^{\varepsilon}(t, r, v) d r d v d t \rightarrow \int_{0}^{T} \int_{0}^{2 \pi} \int_{\mathbb{R}^{2}} F(t, \tau, r, v) \phi(t, \tau, r, v) d r d v d \tau d t,
$$

as $\varepsilon \rightarrow 0$, for all $\phi \in \mathcal{C}^{0}$ where $\mathcal{C}^{i}:=C_{c}^{i}\left([0, T) ; C_{\#}^{i}\left(\mathbb{R} ; C_{c}^{i}\left(\mathbb{R}^{2}\right)\right)\right)$ for $i=0,1$.

Remark 2.1 (Notations). In the above definition and in the remainder of the paper, we introduce the following general notation:

(i) Subscript \# in space definition stands for $2 \pi$-periodicity in $\tau$.

(ii) Bracket $[\cdot]^{\varepsilon}$ stands for $[\phi]^{\varepsilon}(t, r, v):=\phi(t, t / \varepsilon, r, v)$ for any function $\phi$ depending of the four variables $(t, \tau, r, v) \in[0, T) \times \mathbb{R} \times \mathbb{R}^{2}$.

Moreover, in the whole section we will use the 2D-rotation of angle $\tau$ of a two-dimensional vector.

Definition 2.2. For all $\tau \in[0,2 \pi]$ and all $(r, v) \in \mathbb{R}^{2}$, we define the $2 D$-rotation $\mathcal{R}^{\tau}$ of angle $\tau$ by:

$$
\mathcal{R}^{\tau}(r, v)=\left(\begin{array}{cc}
\cos \tau & -\sin \tau \\
\sin \tau & \cos \tau
\end{array}\right)\left(\begin{array}{l}
r \\
v
\end{array}\right)=\left(\begin{array}{c}
r \cos \tau-v \sin \tau \\
r \sin \tau+v \cos \tau
\end{array}\right) .
$$

Its inverse in then given by $\mathcal{R}^{-\tau}$ whose first component is denoted by $\mathcal{R}_{r}^{-\tau}$. 
Remark 2.2. Thanks to the above definition, we have that:

$$
\mathcal{R}^{\tau}(r, v) \cdot\left(\begin{array}{c}
q \\
u
\end{array}\right)=\left(\begin{array}{c}
r \\
v
\end{array}\right) \cdot \mathcal{R}^{-\tau}(q, u)
$$

We are now in position to state our main result.

Theorem 2.1. Let $\left(f^{\varepsilon}\right)_{\varepsilon}$ be a sequence of solutions to Vlasov problem (1.1) with the electric field $E^{\varepsilon}$ given by 1.2 . There exist two functions $F:(t, \tau, r, v) \in[0, T) \times \mathbb{R} \times \mathbb{R}^{2} \mapsto F(t, \tau, r, v)$ and $F_{1}:(t, \tau, r, v) \in[0, T) \times \mathbb{R} \times \mathbb{R}^{2} \mapsto F(t, \tau, r, v), 2 \pi$-periodic with respect to $\tau \in \mathbb{R}$, such that

$$
f^{\varepsilon}(t, r, v) \approx 2 \pi F\left(t, \frac{t}{\varepsilon}, r, v\right)+\varepsilon 2 \pi F_{1}\left(t, \frac{t}{\varepsilon}, r, v\right)
$$

when $\varepsilon$ is small. Moreover, regarding the zeroth order term, there exists a function $G:(t, q, u) \in$ $[0, T) \times \mathbb{R}^{2} \mapsto G(t, q, u)$ such that

$$
F(t, \tau, r, v)=G\left(t, \mathcal{R}^{\tau}(r, v)\right)
$$

where $\mathcal{R}^{\tau}$ is the rotation defined in Definition 2.2 and $G$ is solution to the problem

$$
\left\{\begin{array}{l}
\frac{\partial G}{\partial t}(t, q, u)+\frac{1}{2 \pi} \int_{0}^{2 \pi} \mathcal{R}^{\tau}\left(0, E^{0}\left(t, \tau, \mathcal{R}_{r}^{-\tau}(q, u)\right)\right) d \tau \cdot \nabla_{q, u} G(t, q, u)=0 \\
G(t=0, q, u)=\frac{1}{2 \pi} f_{0}(q, u) .
\end{array}\right.
$$

Regarding the first order term, there exist two functions $G_{1}:(t, q, u) \in[0, T) \times \mathbb{R}^{2} \mapsto G_{1}(t, q, u)$ and $W:(t, \tau, q, u) \in[0, T) \times \mathbb{R} \times \mathbb{R}^{2} \mapsto W(t, \tau, q, u)$ such that

$$
F_{1}(t, \tau, r, v)=G_{1}\left(t, \mathcal{R}^{\tau}(r, v)\right)+W\left(t, \tau, \mathcal{R}^{\tau}(r, v)\right)
$$

where on the one hand, $W$ is given by

$$
\begin{aligned}
W(t, \tau, q, u)= & {\left[\frac{\tau}{2 \pi} \int_{0}^{2 \pi} \mathcal{R}^{\sigma}\left(0, E^{0}\left(t, \sigma, \mathcal{R}_{r}^{-\sigma}(q, u)\right)\right) d \sigma\right.} \\
& \left.-\int_{0}^{\tau} \mathcal{R}^{\sigma}\left(0, E^{0}\left(t, \sigma, \mathcal{R}_{r}^{-\sigma}(q, u)\right)\right) d \sigma\right] \cdot \nabla_{q, u} G(t, q, u),
\end{aligned}
$$


and on the other hand, $G_{1}$ is solution to the problem

$$
\left\{\begin{array}{l}
\frac{\partial G_{1}}{\partial t}(t, q, u)+\frac{1}{2 \pi} \int_{0}^{2 \pi} \mathcal{R}^{\tau}\left(0, E^{0}\left(t, \tau, \mathcal{R}_{r}^{-\tau}(q, u)\right)\right) d \tau \cdot \nabla_{q, u} G_{1}(t, q, u)= \\
\frac{1}{2 \pi} \int_{0}^{2 \pi}\left[\int_{0}^{\tau} \mathcal{R}^{\sigma}\left(0, \frac{\partial E^{0}}{\partial t}\left(t, \sigma, \mathcal{R}_{r}^{-\sigma}(q, u)\right)\right) d \sigma\right. \\
\left.\quad-\frac{\tau}{2 \pi} \int_{0}^{2 \pi} \mathcal{R}^{\sigma}\left(0, \frac{\partial E^{0}}{\partial t}\left(t, \sigma, \mathcal{R}_{r}^{-\sigma}(q, u)\right)\right) d \sigma\right] d \tau \cdot \nabla_{q, u} G(t, q, u) \\
+\left[\frac{1}{4 \pi} \int_{0}^{2 \pi} \nabla_{q, u} \mathcal{R}^{\sigma}\left(0, E^{0}\left(t, \sigma, \mathcal{R}_{r}^{-\sigma}(q, u)\right)\right) d \sigma \int_{0}^{2 \pi} \mathcal{R}^{\sigma}\left(0, E^{0}\left(t, \sigma, \mathcal{R}_{r}^{-\sigma}(q, u)\right)\right) d \sigma\right. \\
\left.\quad-\frac{1}{2 \pi} \int_{0}^{2 \pi}\left(\nabla_{q, u} \mathcal{R}^{\tau}\left(0, E^{0}\left(t, \tau, \mathcal{R}_{r}^{-\tau}(q, u)\right)\right) \int_{0}^{\tau} \mathcal{R}^{\sigma}\left(0, E^{0}\left(t, \sigma, \mathcal{R}_{r}^{-\sigma}(q, u)\right)\right) d \sigma\right) d \tau\right] \\
\cdot \nabla_{q, u} G(t, q, u) d \tau
\end{array}\right]
$$

Remark 2.3.

- Note that the expression of $W$ in (2.8) and problems (2.6) and (2.9) do not depend on $1 / \varepsilon$-frequency oscillations anymore.

- The Two-Scale approximation result in (2.4) will be specified in 2.29 , in the proof of the Theorem, which is given in the two following sections.

\subsection{Zeroth order approximation}

In this section, we briefly recall arguments developed in 2]. Let us consider the Vlasov equation in problem (1.1). First, we multiply by $f^{\varepsilon}$ and integrate over $r \in \mathbb{R}^{+}$and $v \in \mathbb{R}$ to obtain:

$$
\frac{1}{2} \frac{d\left(\left\|f^{\varepsilon}(t, \cdot, \cdot)\right\|_{L^{2}\left(\mathbb{R}^{2}\right)}^{2}\right)}{d t}=0
$$

which leads to the following estimate:

$$
\left\|f^{\varepsilon}\right\|_{L^{\infty}\left([0, T) ; L^{2}\left(\mathbb{R}^{2}\right)\right)} \leq C .
$$

Then it is known that, up to a subsequence, $\left(f^{\varepsilon}\right)_{\varepsilon}$ Two-Scale converges to some $F:(t, \tau, r, v) \mapsto$ $F(t, \tau, r, v)$ with $F \in L^{\infty}\left([0, T) ; L_{\#}^{\infty}\left(\mathbb{R}, L^{2}\left(\mathbb{R}^{2}\right)\right)\right)$. Now, we multiply the Vlasov equation in (1.1) by $[\phi]^{\varepsilon}$ with $\phi \in \mathcal{C}^{1}$, integrate over $t \in[0, T)$ and $(r, v) \in \mathbb{R}^{2}$ and then integrate by parts. Thus we show that $F$ satisfies in a weak sense the following equation

$$
\frac{\partial F}{\partial \tau}+\left(\begin{array}{c}
v \\
-r
\end{array}\right) \cdot \nabla_{r, v} F=0
$$

where we note that $\varepsilon$ is not involved anymore. We deduce from 2.12 that $F$ is constant along the characteristics associated to this advection equation, and from Theorem 4.2 in 2 that there exists a function $G:(t, q, u) \in[0, T) \times \mathbb{R}^{2} \mapsto G(t, q, u)$ such that 2.5 is satisfied and such that $G$ is solution to problem 2.6 of Theorem 2.1. 
Remark 2.4. Let $G:(t, q, u) \in[0, T) \times \mathbb{R}^{2} \mapsto G(t, q, u)$ be a given function and define the family $\left(F^{\varepsilon}\right)_{\varepsilon>0} b y$

Then

$$
[F]^{\varepsilon}(t, r, v)=G\left(t, \mathcal{R}^{t / \varepsilon}(r, v)\right) .
$$

(i) $\nabla_{r, v}[F]^{\varepsilon}(t, r, v)=\mathcal{R}^{-t / \varepsilon}\left(\nabla_{q, u} G\left(t, \mathcal{R}^{t / \varepsilon}(r, v)\right)\right)$.

(ii) $\frac{\partial[F]^{\varepsilon}}{\partial t}(t, r, v)+\frac{1}{\varepsilon}\left(\begin{array}{c}v \\ -r\end{array}\right) \cdot \nabla_{r, v}[F]^{\varepsilon}(t, r, v)=\frac{\partial G}{\partial t}\left(t, \mathcal{R}^{t / \varepsilon}(r, v)\right)$.

\subsection{First order approximation}

We consider the zeroth order Two-Scale approximation $F$ of $f^{\varepsilon}$ introduced in Section 2.1. Let us define $[F]^{\varepsilon}$ as in Remark 2.1(ii). Then thanks to Remark 2.4(ii)

$$
\frac{\partial[F]^{\varepsilon}}{\partial t}(t, r, v)+\frac{1}{\varepsilon}\left(\begin{array}{c}
v \\
-r
\end{array}\right) \cdot \nabla_{r, v}[F]^{\varepsilon}(t, r, v)=\frac{\partial G}{\partial t}\left(t, \mathcal{R}^{t / \varepsilon}(r, v)\right) .
$$

For the sake of simplicity in computations below, we introduce the notation $E^{\varepsilon}=E_{0}(t, t / \varepsilon, r)+$ $\varepsilon E_{1}(t, t / \varepsilon, r)$ and omit the dependency of $[F]^{\varepsilon}$ and its partial derivatives in $(t, r, v)$. First, we add the term $E^{\varepsilon} \partial[F]^{\varepsilon} / \partial v$ on both sides of equation (2.14) to obtain (2.15). Then we rewrite the right-hand side to show up the term $\nabla_{r, v}[F]^{\varepsilon}$ and get 2.16$)$. Using Remark 2.4 (i) and Remark 2.2, we obtain 2.17 and 2.18 respectively.

$$
\begin{aligned}
\frac{\partial[F]^{\varepsilon}}{\partial t} & +\frac{1}{\varepsilon}\left(\begin{array}{c}
v \\
-r
\end{array}\right) \cdot \nabla_{r, v}[F]^{\varepsilon}+E^{\varepsilon} \frac{\partial[F]^{\varepsilon}}{\partial v}= \\
& =E^{\varepsilon} \frac{\partial[F]^{\varepsilon}}{\partial v}+\frac{\partial G}{\partial t}\left(t, \mathcal{R}^{t / \varepsilon}(r, v)\right), \\
& =\left(\begin{array}{c}
0 \\
E^{\varepsilon}
\end{array}\right) \cdot \nabla_{r, v}[F]^{\varepsilon}+\frac{\partial G}{\partial t}\left(t, \mathcal{R}^{t / \varepsilon}(r, v)\right), \\
& =\left(\begin{array}{c}
0 \\
E^{\varepsilon}
\end{array}\right) \cdot \mathcal{R}^{-t / \varepsilon}\left(\nabla_{q, u} G\left(t, \mathcal{R}^{t / \varepsilon}(r, v)\right)\right)+\frac{\partial G}{\partial t}\left(t, \mathcal{R}^{t / \varepsilon}(r, v)\right), \\
& =\mathcal{R}^{t / \varepsilon}\left(0, E^{\varepsilon}\right) \cdot \nabla_{q, u} G\left(t, \mathcal{R}^{t / \varepsilon}(r, v)\right)+\frac{\partial G}{\partial t}\left(t, \mathcal{R}^{t / \varepsilon}(r, v)\right) .
\end{aligned}
$$

To handle the right-hand side of 2.18 , we introduce the function $\Upsilon^{\varepsilon}$ such that

$$
\Upsilon^{\varepsilon}(t, \tau, r, v):=\mathcal{R}^{\tau}\left(0, E^{\varepsilon}\right) \cdot \nabla_{q, u} G\left(t, \mathcal{R}^{\tau}(r, v)\right)+\frac{\partial G}{\partial t}\left(t, \mathcal{R}^{\tau}(r, v)\right) .
$$

Then defining $\left[\Upsilon^{\varepsilon}\right]^{\varepsilon}$ as in Remark 2.1(ii), we can subtract 2.18) from 1.1) and multiply the result by $1 / \varepsilon$ to obtain

$$
\frac{\partial}{\partial t}\left(\frac{f^{\varepsilon}-[F]^{\varepsilon}}{\varepsilon}\right)+\frac{1}{\varepsilon}\left(\begin{array}{c}
v \\
-r
\end{array}\right) \cdot \nabla_{r, v}\left(\frac{f^{\varepsilon}-[F]^{\varepsilon}}{\varepsilon}\right)+E^{\varepsilon} \frac{\partial}{\partial v}\left(\frac{f^{\varepsilon}-[F]^{\varepsilon}}{\varepsilon}\right)=-\frac{1}{\varepsilon}\left[\Upsilon^{\varepsilon}\right]^{\varepsilon} .
$$

Now, let $W^{\varepsilon}:(t, \tau, q, u) \in[0, T) \times \mathbb{R} \times \mathbb{R}^{2} \mapsto W^{\varepsilon}(t, \tau, q, u)$ be such that

$$
\frac{\partial W^{\varepsilon}}{\partial \tau}\left(t, \tau, \mathcal{R}^{\tau}(r, v)\right)=-\Upsilon^{\varepsilon}(t, \tau, r, v)
$$

On the one hand, if we substitute $\Upsilon^{\varepsilon}$ by its expression in 2.19 and then write equation 2.21 in variables $(q, u)=\mathcal{R}^{\tau}(r, v)$, we obtain

$$
\frac{\partial W^{\varepsilon}}{\partial \tau}(t, \tau, q, u)=-\mathcal{R}^{\tau}\left(0, E^{\varepsilon}\left(t, \tau, \mathcal{R}_{r}^{-\tau}(q, u)\right)\right) \cdot \nabla_{q, u} G(t, q, u)-\frac{\partial G}{\partial t}(t, q, u) .
$$


We substitute the time derivative of $G$ in the right-hand side of 2.22 by its expression in 2.6 . Integrating the result with respect to the second variable from 0 to $\tau$ gives

$$
\begin{aligned}
W^{\varepsilon}(t, \tau, q, u)= & {\left[\frac{\tau}{2 \pi} \int_{0}^{2 \pi} \mathcal{R}^{\sigma}\left(0, E^{0}\left(t, \sigma, \mathcal{R}_{r}^{-\sigma}(q, u)\right)\right) d \sigma\right.} \\
& \left.-\int_{0}^{\tau} \mathcal{R}^{\tau}\left(0, E^{0}\left(t, \tau, \mathcal{R}_{r}^{-\tau}(q, u)\right)\right) d \sigma\right] \cdot \nabla_{q, u} G(t, q, u) \\
& -\varepsilon \int_{0}^{\tau} \mathcal{R}^{\tau}\left(0, E^{1}\left(t, \tau, \mathcal{R}_{r}^{-\tau}(q, u)\right)\right) d \sigma \cdot \nabla_{q, u} G(t, q, u) .
\end{aligned}
$$

When $\varepsilon$ goes to 0 in 2.23 , we obtain the limit $W$ of $W^{\varepsilon}$ defined by 2.8 in Theorem 2.1 .

On the other hand, let us introduce $\widetilde{W}^{\varepsilon}$ define by $\widetilde{W}^{\varepsilon}(t, \tau, r, v):=W^{\varepsilon}\left(t, \tau, \mathcal{R}^{\tau}(r, v)\right)$. Therefore, 2.21 is equivalent to

$$
\frac{\partial \widetilde{W}^{\varepsilon}}{\partial \tau}(t, \tau, r, v)+\left(\begin{array}{c}
v \\
-r
\end{array}\right) \cdot \nabla_{r, v} \widetilde{W}^{\varepsilon}(t, \tau, r, v)=-\Upsilon^{\varepsilon}(t, \tau, r, v)
$$

Using Remark 2.1(ii), we note that $\partial_{t}\left[\widetilde{W}^{\varepsilon}\right]^{\varepsilon}=\left[\partial_{t} \widetilde{W}^{\varepsilon}\right]^{\varepsilon}+\left[\partial_{\tau} \widetilde{W}^{\varepsilon}\right]^{\varepsilon} / \varepsilon$ and $\partial_{r, v}\left[\widetilde{W^{\varepsilon}}\right]^{\varepsilon}=\left[\partial_{r, v} \widetilde{W}^{\varepsilon}\right]^{\varepsilon}$, and thus, writing 2.24 in $\tau=t / \varepsilon$ and multiplying the result by $1 / \varepsilon$ leads to

$$
\frac{\partial\left[\widetilde{W}^{\varepsilon}\right]^{\varepsilon}}{\partial t}-\left[\frac{\partial \widetilde{W^{\varepsilon}}}{\partial t}\right]^{\varepsilon}+\frac{1}{\varepsilon}\left(\begin{array}{c}
v \\
-r
\end{array}\right) \cdot \nabla_{r, v}\left[\widetilde{W}^{\varepsilon}\right]^{\varepsilon}=-\frac{1}{\varepsilon}\left[\Upsilon^{\varepsilon}\right]^{\varepsilon} .
$$

Now, we subtract 2.25) from (2.20) and subtract from the both sides of the result the term $E^{\varepsilon} \partial_{v}\left[\widetilde{W}^{\varepsilon}\right]^{\varepsilon}$ to obtain

$$
\begin{aligned}
\frac{\partial}{\partial t}\left(\frac{f^{\varepsilon}-[F]^{\varepsilon}}{\varepsilon}-\left[\widetilde{W}^{\varepsilon}\right]^{\varepsilon}\right) & +\frac{1}{\varepsilon}\left(\begin{array}{c}
v \\
-r
\end{array}\right) \cdot \nabla_{r, v}\left(\frac{f^{\varepsilon}-[F]^{\varepsilon}}{\varepsilon}-\left[\widetilde{W}^{\varepsilon}\right]^{\varepsilon}\right) \\
& +E^{\varepsilon} \frac{\partial}{\partial v}\left(\frac{f^{\varepsilon}-[F]^{\varepsilon}}{\varepsilon}-\left[\widetilde{\left.W^{\varepsilon}\right]^{\varepsilon}}\right)\right. \\
& =-\left[\frac{\partial \widetilde{W}^{\varepsilon}}{\partial t}\right]^{\varepsilon}-E^{\varepsilon} \frac{\partial\left[\widetilde{W}^{\varepsilon}\right]^{\varepsilon}}{\partial v} .
\end{aligned}
$$

In the following, we want to obtain an a priori estimate, in order to prove our convergence result. Therefore, we multiply $(2.26)$ by $\frac{f^{\varepsilon}-[F]^{\varepsilon}}{\varepsilon}-\left[\widetilde{W}^{\varepsilon}\right]^{\varepsilon}$ and then integrate by parts. Consequently,

$$
\begin{aligned}
\frac{1}{2} \frac{d}{d t} \| \frac{f^{\varepsilon}-[F]^{\varepsilon}}{\varepsilon} & -\left[\widetilde{\left.W^{\varepsilon}\right]^{\varepsilon}} \|_{L^{2}\left(\mathbb{R}^{2}\right)}^{2}(t)\right. \\
\leq & \left\|\left[\frac{\partial \widetilde{W^{\varepsilon}}}{\partial t}\right]^{\varepsilon}+\left(\begin{array}{c}
0 \\
E^{\varepsilon}
\end{array}\right) \cdot \nabla_{r, v}\left[\widetilde{W^{\varepsilon}}\right]^{\varepsilon}\right\|_{L^{2}\left(\mathbb{R}^{2}\right)}(t)\left\|\frac{f^{\varepsilon}-[F]^{\varepsilon}}{\varepsilon}-\left[\widetilde{W^{\varepsilon}}\right]^{\varepsilon}\right\|_{L^{2}\left(\mathbb{R}^{2}\right)}
\end{aligned}
$$

If the first factor on the right-hand side of 2.27$)$ is bounded, by Gronwall Lemma, we obtain the following estimate

$$
\left\|\frac{f^{\varepsilon}-[F]^{\varepsilon}}{\varepsilon}-\left[\widetilde{W}^{\varepsilon}\right]^{\varepsilon}\right\|_{L^{\infty}\left([0, T) ; L^{2}\left(\mathbb{R}^{2}\right)\right)} \leq C .
$$


From this estimate, we deduce the following Two-Scale convergence result

$$
\frac{f^{\varepsilon}-[F]^{\varepsilon}}{\varepsilon}-\left[\widetilde{W}^{\varepsilon}\right]^{\varepsilon} \longrightarrow F_{1}-\widetilde{W}
$$

where $\widetilde{W}=W\left(t, \tau, \mathcal{R}^{\tau}(r, v)\right)$ and $W$ is given by $(2.8)$.

Now, we want to compute $F_{1}$. Returning to (2.26), we multiply by $\phi \in \mathcal{C}^{1}$ (see Definition 2.1), integrate over $t \in[0, T)$ and $(r, v) \in \mathbb{R}^{2}$ and then integrate by parts. We obtain

$$
\begin{array}{r}
-\int_{0}^{T} \int_{\mathbb{R}^{2}}\left(\frac{f^{\varepsilon}-[F]^{\varepsilon}}{\varepsilon}-\left[\widetilde{W}^{\varepsilon}\right]^{\varepsilon}\right)\left(\left[\frac{\partial \phi}{\partial t}\right]^{\varepsilon}+E^{\varepsilon}\left[\frac{\partial \phi}{\partial v}\right]^{\varepsilon}\right) d r d v d t \\
-\frac{1}{\varepsilon} \int_{0}^{T} \int_{\mathbb{R}^{2}}\left(\frac{f^{\varepsilon}-[F]^{\varepsilon}}{\varepsilon}-\left[\widetilde{W}^{\varepsilon}\right]^{\varepsilon}\right)\left(\left[\frac{\partial \phi}{\partial \tau}\right]^{\varepsilon}+\left(\begin{array}{c}
v \\
-r
\end{array}\right) \cdot\left[\nabla_{r, v} \phi\right]^{\varepsilon}\right) d r d v d t \\
=-\int_{0}^{T} \int_{\mathbb{R}^{2}}\left(\left[\frac{\partial \widetilde{W}^{\varepsilon}}{\partial t}\right]^{\varepsilon}-\left(\begin{array}{c}
0 \\
E^{\varepsilon}
\end{array}\right) \cdot\left[\nabla_{r, v} \widetilde{W}^{\varepsilon}\right]^{\varepsilon}\right)[\phi]^{\varepsilon} d r d v d t .
\end{array}
$$

Multiplying (2.30) by $\varepsilon$ and passing to the limit using the Two-Scale convergence 2.29), we obtain

$$
\frac{\partial}{\partial \tau}\left(F_{1}-\widetilde{W}\right)+\left(\begin{array}{c}
v \\
-r
\end{array}\right) \cdot \nabla_{r, v}\left(F_{1}-\widetilde{W}\right)=0
$$

in a weak sense. Thus, $F_{1}-\widetilde{W}$ is constant along the characteristics, hence there exists a function $G_{1}:(t, q, u) \in[0, T) \times \mathbb{R}^{2} \mapsto G_{1}(t, q, u)$ such that

$$
F_{1}(t, \tau, r, v)-\widetilde{W}(t, \tau, r, v)=G_{1}\left(t, \mathcal{R}^{\tau}(r, v)\right)
$$

and therefore, thanks to the definition of $\widetilde{W}$, we obtain the decomposition (2.7) of $F_{1}$ in Theorem 2.1 Now, let $\gamma:(t, q, u) \in[0, T) \times \mathbb{R}^{2} \mapsto \gamma(t, q, u)$ be a function in $C_{c}^{1}\left([0, T) ; C_{c}^{1}\left(\mathbb{R}^{2}\right)\right)$ such that

$$
\phi(t, \tau, r, v)=\gamma\left(t, \mathcal{R}^{\tau}(r, v)\right)
$$

When considering (2.30) for this function $\phi$, the second term on the left-hand side vanishes, and we obtain

$$
\begin{aligned}
& \int_{0}^{T} \int_{\mathbb{R}^{2}}\left(\frac{f^{\varepsilon}-[F]^{\varepsilon}}{\varepsilon}-\left[\widetilde{W}^{\varepsilon}\right]^{\varepsilon}\right)\left(\left[\frac{\partial \phi}{\partial t}\right]^{\varepsilon}+E^{\varepsilon}\left[\frac{\partial \phi}{\partial v}\right]^{\varepsilon}\right) d r d v d t \\
& =-\int_{0}^{T} \int_{\mathbb{R}^{2}}\left(\left[\frac{\partial \widetilde{W}^{\varepsilon}}{\partial t}\right]^{\varepsilon}-\left(\begin{array}{c}
0 \\
E^{\varepsilon}
\end{array}\right) \cdot\left[\nabla_{r, v} \widetilde{W}^{\varepsilon}\right]^{\varepsilon}\right)[\phi]^{\varepsilon} d r d v d t
\end{aligned}
$$

Thanks to the Two-Scale convergence, we pass to the limit as $\varepsilon \rightarrow 0$ and integrate over $\tau \in[0,2 \pi]$ which leads to

$$
\begin{array}{r}
\int_{0}^{T} \int_{0}^{2 \pi} \int_{\mathbb{R}^{2}} G_{1}\left(t, \mathcal{R}^{\tau}(r, v)\right)\left(\frac{\partial \phi}{\partial t}+\left(\begin{array}{c}
0 \\
E^{0}
\end{array}\right) \cdot \nabla_{r, v} \phi\right) d r d v d \tau d t \\
=\int_{0}^{T} \int_{0}^{2 \pi} \int_{\mathbb{R}^{2}}\left(\frac{\partial \widetilde{W}}{\partial t}-\left(\begin{array}{c}
0 \\
E^{0}
\end{array}\right) \cdot \nabla_{r, v} \widetilde{W}\right) \phi d r d v d \tau d t
\end{array}
$$


Then, substituting $\phi$ by its expression in terms of $\gamma$ and changing the variables $(r, v)$ in $(q, u)=$ $\mathcal{R}^{\tau}(r, v)$ give

$$
\begin{aligned}
\int_{0}^{T} \int_{0}^{2 \pi} \int_{\mathbb{R}^{2}} G_{1}(t, q, u)( & \frac{\partial \gamma}{\partial t}(t, q, u) \\
& \left.+\mathcal{R}^{\tau}\left(0, E^{0}\left(t, \tau, \mathcal{R}_{r}^{-\tau}(q, u)\right)\right) \cdot \nabla_{q, u} \gamma(t, q, u)\right) d q d u d \tau d t \\
=\int_{0}^{T} \int_{0}^{2 \pi} \int_{\mathbb{R}^{2}}( & \frac{\partial W}{\partial t}(t, \tau, q, u) \\
& \left.+\mathcal{R}^{\tau}\left(0, E^{0}\left(t, \tau, \mathcal{R}_{r}^{-\tau}(q, u)\right)\right) \cdot \nabla_{q, u} W(t, \tau, q, u)\right) \gamma(t, q, u) d q d u d \tau d t
\end{aligned}
$$

We finally deduce that $G_{1}$ satisfies, in a weak sense, the following problem

$$
\left\{\begin{array}{l}
\frac{\partial G_{1}}{\partial t}(t, q, u)+\frac{1}{2 \pi} \int_{0}^{2 \pi} \mathcal{R}^{\tau}\left(0, E^{0}\left(t, \tau, \mathcal{R}_{r}^{-\tau}(q, u)\right)\right) d \tau \cdot \nabla_{q, u} G_{1}(t, q, u)= \\
-\frac{1}{2 \pi} \int_{0}^{2 \pi}\left(\frac{\partial W}{\partial t}(t, \tau, q, u)+\mathcal{R}^{\tau}\left(0, E^{0}\left(t, \tau, \mathcal{R}_{r}^{-\tau}(q, u)\right)\right) \cdot \nabla_{q, u} W(t, \tau, q, u)\right) d \tau \\
G_{1}(t=0, q, u)=0 .
\end{array}\right.
$$

To handle the right-hand side of (2.37), we need to compute the partial derivatives of $W$. First, from (2.8), we have that

$$
\begin{aligned}
\frac{\partial W}{\partial t}(t, \tau, q, u)= & {\left[\frac{\tau}{2 \pi} \int_{0}^{2 \pi} \mathcal{R}^{\sigma}\left(0, \frac{\partial E^{0}}{\partial t}\left(t, \sigma, \mathcal{R}_{r}^{-\sigma}(q, u)\right)\right) d \sigma\right.} \\
& \left.-\int_{0}^{\tau} \mathcal{R}^{\sigma}\left(0, \frac{\partial E^{0}}{\partial t}\left(t, \sigma, \mathcal{R}_{r}^{-\sigma}(q, u)\right)\right) d \sigma\right] \cdot \nabla_{q, u} G(t, q, u) \\
+ & {\left[\frac{\tau}{2 \pi} \int_{0}^{2 \pi} \mathcal{R}^{\sigma}\left(0, E^{0}\left(t, \sigma, \mathcal{R}_{r}^{-\sigma}(q, u)\right)\right) d \sigma\right.} \\
& \left.-\int_{0}^{\tau} \mathcal{R}^{\sigma}\left(0, E^{0}\left(t, \sigma, \mathcal{R}_{r}^{-\sigma}(q, u)\right)\right) d \sigma\right] \cdot \nabla_{q, u} \frac{\partial G}{\partial t}(t, q, u),
\end{aligned}
$$

so that we can then replace $\partial_{t} G$ by its expression in 2.6 to get

$$
\begin{aligned}
\frac{\partial W}{\partial t}(t, \tau, q, u)= & {\left[\frac{\tau}{2 \pi} \int_{0}^{2 \pi} \mathcal{R}^{\sigma}\left(0, \frac{\partial E^{0}}{\partial t}\left(t, \sigma, \mathcal{R}_{r}^{-\sigma}(q, u)\right)\right) d \sigma\right.} \\
& \left.-\int_{0}^{\tau} \mathcal{R}^{\sigma}\left(0, \frac{\partial E^{0}}{\partial t}\left(t, \sigma, \mathcal{R}_{r}^{-\sigma}(q, u)\right)\right) d \sigma\right] \cdot \nabla_{q, u} G(t, q, u) \\
+ & {\left[\frac{\tau}{2 \pi} \int_{0}^{2 \pi} \mathcal{R}^{\sigma}\left(0, E^{0}\left(t, \sigma, \mathcal{R}_{r}^{-\sigma}(q, u)\right)\right) d \sigma\right.} \\
& \left.-\int_{0}^{\tau} \mathcal{R}^{\sigma}\left(0, E^{0}\left(t, \sigma, \mathcal{R}_{r}^{-\sigma}(q, u)\right)\right) d \sigma\right] \cdot \\
& \cdot\left[\left[\frac{1}{2 \pi} \int_{0}^{2 \pi} \nabla_{q, u} \mathcal{R}^{\sigma}\left(0, E^{0}\left(t, \sigma, \mathcal{R}_{r}^{-\sigma}(q, u)\right)\right) d \sigma\right] \nabla_{q, u} G(t, q, u)\right. \\
& \left.+H_{q, u} G(t, q, u) \frac{1}{2 \pi} \int_{0}^{2 \pi} \mathcal{R}^{\sigma}\left(0, E^{0}\left(t, \sigma, \mathcal{R}_{r}^{-\sigma}(q, u)\right)\right) d \sigma\right]
\end{aligned}
$$


where $H_{q, u}$ is the Hessian matrix with respect to variable $q$ and $u$. Next, from 2.8, we deduce that

$$
\begin{aligned}
\nabla_{q, u} W(t, \tau, q, u)= & {\left[\frac{\tau}{2 \pi} \int_{0}^{2 \pi} \nabla_{q, u} \mathcal{R}^{\sigma}\left(0, E^{0}\left(t, \sigma, \mathcal{R}_{r}^{-\sigma}(q, u)\right)\right) d \sigma\right.} \\
& \left.-\int_{0}^{\tau} \nabla_{q, u} \mathcal{R}^{\sigma}\left(0, E^{0}\left(t, \sigma, \mathcal{R}_{r}^{-\sigma}(q, u)\right)\right) d \sigma\right] \cdot \nabla_{q, u} G(t, q, u) \\
+ & H_{q, u} G(t, q, u)\left[\frac{\tau}{2 \pi} \int_{0}^{2 \pi} \mathcal{R}^{\sigma}\left(0, E^{0}\left(t, \sigma, \mathcal{R}_{r}^{-\sigma}(q, u)\right)\right) d \sigma\right. \\
& \left.-\int_{0}^{\tau} \mathcal{R}^{\sigma}\left(0, E^{0}\left(t, \sigma, \mathcal{R}_{r}^{-\sigma}(q, u)\right)\right) d \sigma\right]
\end{aligned}
$$

Then, we take the scalar product of 2.40 with vector $\mathcal{R}^{\tau}\left(0, E^{0}\left(t, \tau, \mathcal{R}_{r}^{-\tau}(q, u)\right)\right)$, sum the result with 2.39 , and then integrate over $\tau \in[0,2 \pi]$. Using Fubini's Theorem for the terms involving the Hessian matrix of $G$, which is symmetric, yields

$$
\begin{aligned}
& \int_{0}^{2 \pi}\left(\frac{\partial W}{\partial t}(t, \tau, q, u)+\mathcal{R}^{\tau}\left(0, E^{0}\left(t, \tau, \mathcal{R}_{r}^{-\tau}(q, u)\right)\right) \cdot \nabla_{q, u} W(t, \tau, q, u)\right) d \tau \\
& =\int_{0}^{2 \pi}\left[\frac{\tau}{2 \pi} \int_{0}^{2 \pi} \mathcal{R}^{\sigma}\left(0, \frac{\partial E^{0}}{\partial t}\left(t, \sigma, \mathcal{R}_{r}^{-\sigma}(q, u)\right)\right) d \sigma\right. \\
& \left.-\int_{0}^{\tau} \mathcal{R}^{\sigma}\left(0, \frac{\partial E^{0}}{\partial t}\left(t, \sigma, \mathcal{R}_{r}^{-\sigma}(q, u)\right)\right) d \sigma\right] \cdot \nabla_{q, u} G(t, q, u) d \tau \\
& +\int_{0}^{2 \pi}\left[\left[\frac{\tau}{2 \pi} \int_{0}^{2 \pi} \nabla_{q, u} \mathcal{R}^{\sigma}\left(0, E^{0}\left(t, \sigma, \mathcal{R}_{r}^{-\sigma}(q, u)\right)\right) d \sigma\right.\right. \\
& \left.-\int_{0}^{\tau} \nabla_{q, u} \mathcal{R}^{\sigma}\left(0, E^{0}\left(t, \sigma, \mathcal{R}_{r}^{-\sigma}(q, u)\right)\right) d \sigma\right] \mathcal{R}^{\tau}\left(0, E^{0}\left(t, \tau, \mathcal{R}_{r}^{-\tau}(q, u)\right)\right) \\
& -\frac{1}{2 \pi} \int_{0}^{2 \pi} \nabla_{q, u} \mathcal{R}^{\sigma}\left(0, E^{0}\left(t, \sigma, \mathcal{R}_{r}^{-\sigma}(q, u)\right)\right) d \sigma \\
& {\left[\frac{\tau}{2 \pi} \int_{0}^{2 \pi} \mathcal{R}^{\sigma}\left(0, E^{0}\left(t, \sigma, \mathcal{R}_{r}^{-\sigma}(q, u)\right)\right) d \sigma\right.} \\
& \left.\left.-\int_{0}^{\tau} \mathcal{R}^{\sigma}\left(0, E^{0}\left(t, \sigma, \mathcal{R}_{r}^{-\sigma}(q, u)\right)\right) d \sigma\right]\right] \cdot \nabla_{q, u} G(t, q, u) d \tau \\
& -\int_{0}^{2 \pi} H_{q, u} G(t, q, u)\left[\frac{\tau}{2 \pi} \int_{0}^{2 \pi} \mathcal{R}^{\sigma}\left(0, E^{0}\left(t, \sigma, \mathcal{R}_{r}^{-\sigma}(q, u)\right)\right) d \sigma\right. \\
& \left.-\int_{0}^{\tau} \mathcal{R}^{\sigma}\left(0, E^{0}\left(t, \sigma, \mathcal{R}_{r}^{-\sigma}(q, u)\right)\right) d \sigma\right] . \\
& \cdot\left[\frac{1}{2 \pi} \int_{0}^{2 \pi} \mathcal{R}^{\sigma}\left(0, E^{0}\left(t, \sigma, \mathcal{R}_{r}^{-\sigma}(q, u)\right)\right) d \sigma\right. \\
& \left.-\mathcal{R}^{\tau}\left(0, E^{0}\left(t, \tau, \mathcal{R}_{r}^{-\tau}(q, u)\right)\right)\right] d \tau .
\end{aligned}
$$

Concerning the two last factors on the right-hand side of (2.41), we notice that the second one is the exact derivative of the first one with respect to $\tau$. Since the Hessian matrix is symmetric and does not depend on $\tau$, we can take it out of the integral. Thus, the remaining integrand is the exact derivative of a quadratic form with respect to variable $\tau$, so that integrating over a period in $\tau$ gives 0. Moreover, we can perform an integration by parts in the first part of the second term on the 
right-hand side of 2.41). We obtain

$$
\begin{aligned}
& \int_{0}^{2 \pi}\left(\frac{\partial W}{\partial t}(t, \tau, q, u)+\mathcal{R}^{\tau}\left(0, E^{0}\left(t, \tau, \mathcal{R}_{r}^{-\tau}(q, u)\right)\right) \cdot \nabla_{q, u} W(t, \tau, q, u)\right) d \tau \\
& =\int_{0}^{2 \pi}\left[\frac{\tau}{2 \pi} \int_{0}^{2 \pi} \mathcal{R}^{\sigma}\left(0, \frac{\partial E^{0}}{\partial t}\left(t, \sigma, \mathcal{R}_{r}^{-\sigma}(q, u)\right)\right) d \sigma\right. \\
& \left.-\int_{0}^{\tau} \mathcal{R}^{\sigma}\left(0, \frac{\partial E^{0}}{\partial t}\left(t, \sigma, \mathcal{R}_{r}^{-\sigma}(q, u)\right)\right) d \sigma\right] \cdot \nabla_{q, u} G(t, q, u) d \tau \\
& -\int_{0}^{2 \pi}\left[\left[\frac{1}{2 \pi} \int_{0}^{2 \pi} \nabla_{q, u} \mathcal{R}^{\sigma}\left(0, E^{0}\left(t, \sigma, \mathcal{R}_{r}^{-\sigma}(q, u)\right)\right) d \sigma\right.\right. \\
& \left.-\nabla_{q, u} \mathcal{R}^{\tau}\left(0, E^{0}\left(t, \tau, \mathcal{R}_{r}^{-\tau}(q, u)\right)\right)\right] \int_{0}^{\tau} \mathcal{R}^{\sigma}\left(0, E^{0}\left(t, \sigma, \mathcal{R}_{r}^{-\sigma}(q, u)\right)\right) d \sigma \\
& -\frac{1}{2 \pi} \int_{0}^{2 \pi} \nabla_{q, u} \mathcal{R}^{\sigma}\left(0, E^{0}\left(t, \sigma, \mathcal{R}_{r}^{-\sigma}(q, u)\right)\right) d \sigma \\
& {\left[\frac{\tau}{2 \pi} \int_{0}^{2 \pi} \mathcal{R}^{\sigma}\left(0, E^{0}\left(t, \sigma, \mathcal{R}_{r}^{-\sigma}(q, u)\right)\right) d \sigma\right.} \\
& \left.\left.-\int_{0}^{\tau} \mathcal{R}^{\sigma}\left(0, E^{0}\left(t, \sigma, \mathcal{R}_{r}^{-\sigma}(q, u)\right)\right) d \sigma\right]\right] \cdot \nabla_{q, u} G(t, q, u) d \tau .
\end{aligned}
$$

In the second term on the right-hand side of 2.42 , we remark that first and last products cancel. Substituting (2.42) in the right-hand side of the equation in (2.37) finally leads to problem $(2.9)$.

\section{NumERICAL ALGORITHM}

To build our numerical algorithm, we use a Particle-in-Cell (PIC) method, that deals with macroparticles rather than directly with the distribution function.

Theorem 2.1 states that, provided that problem 2.6 is solved and space derivatives of $G$ can be computed, we can compute $W$ from (2.8) and the source term in problem (2.9). Then it remains to solve problem $(2.9)$ to obtain $G_{1}$. From $G, G_{1}$ and $W$, we finally get the Two-Scale first order approximation of $f^{\varepsilon}$ thanks to $(2.4),(2.5)$ and $(2.7)$. Thus, the main steps of the algorithm are the following.

\section{Main steps of the algorithm}

(i) Compute a particle approximation of $G$ and push the macroparticles with respect to the advection operator associated to the partial differential equation in problem $\sqrt[2.6]{ }$, as in 2 .

(ii) Compute an approximation of the gradient $\nabla_{q, u} G$ from the particle approximation of $G$ which is compatible with the desired particle approximation of $\mathrm{W}$.

(iii) Compute a particle approximation of $W$ from equation (2.8).

(iv) Compute a particle approximation of the source term of the partial differential equation in problem 2.9 , and then a particle approximation of $G_{1}$ by solving problem $(2.9)$ with the same advection operator as in problem 2.6.

To deal with the first step, we introduce the following particle approximation of function $G$

$$
G(t, q, u)=\sum_{k=1}^{N_{p}} \omega_{k} \delta\left(q-Q_{k}(t)\right) \delta\left(u-U_{k}(t)\right)
$$


where $\delta$ is the Dirac mass, $N_{p}$ is the number of macroparticles and $\left(Q_{k}(t), U_{k}(t)\right)$ is the position in phase space of macroparticle $k$ which moves along a characteristic curve of the equation in (2.6). Hence our problem is reduced to compute the location of macroparticles at the next time step from their positions at the previous time step, as the solution of the following dynamical system

$$
\frac{d}{d t}\left(\begin{array}{c}
Q_{k} \\
U_{k}
\end{array}\right)=\frac{1}{2 \pi} \int_{0}^{2 \pi} \mathcal{R}^{\tau}\left(0, E^{0}\left(t, \tau, \mathcal{R}_{r}^{-\tau}\left(Q_{k}, U_{k}\right)\right)\right) d \tau
$$

The main difficulty to solve the coupled problem 3.2 lies in the fact that we need to approximate the integral term. A solution to this problem is described in Section 5.1 of [2].

Once the macroparticles are pushed along the characteristics, we can compute $G$ at the next time step. Applying the ad hoc rotation, we then obtain a particle approximation of $F$ from 2.5). Finally, evaluating this approximation in $\tau=t / \varepsilon$ gives the zeroth order approximation of $f^{\varepsilon}$.

For the second step of the algorithm, we need to recover an approximation of the space derivatives of $G$ thanks to the particle approximation of $G$. Therefore, a regularization of approximation (3.1) is needed. To this purpose, we introduce a regular function $\gamma^{\alpha}$ with support included in the interval $[0, \alpha]$, and such that the function $\gamma_{k}^{\alpha}:(q, u) \mapsto \gamma^{\alpha}\left(\sqrt{\left(q-Q_{k}(t)\right)^{2}+\left(u-U_{k}(t)\right)^{2}}\right)$ has an integral with worth 1 . Thus a regularization of (3.1) is given by

$$
G(t, q, u)=\sum_{k=1}^{N_{p}} \omega_{k} \gamma^{\alpha}\left(\sqrt{\left(q-Q_{k}(t)\right)^{2}+\left(u-U_{k}(t)\right)^{2}}\right) .
$$

Then we can compute numerical approximations of the space derivatives of G.

Now we can handle the third step of the algorithm. Indeed, in 2.8), we can compute an approximation of the right-hand side which has the following shape

$$
\sum_{k=1}^{N_{p}} \beta_{k}(t, \tau, q, u)\left(\gamma^{\alpha}\right)^{\prime}\left(\sqrt{\left(q-Q_{k}(t)\right)^{2}+\left(u-U_{k}(t)\right)^{2}}\right) .
$$

In order to get $W$, we need to distribute the above approximation over the macroparticles. Let the particle approximation of $W$ be given by

$$
W(t, \tau, q, u)=\sum_{k=1}^{N_{p}} \widetilde{\beta}_{k}(t, \tau) \delta\left(q-Q_{k}(t)\right) \delta\left(u-U_{k}(t)\right),
$$

which can be regularized by

$$
W(t, \tau, q, u)=\sum_{k=1}^{N_{p}} \widetilde{\beta}_{k}(t, \tau) \gamma^{\alpha}\left(\sqrt{\left(q-Q_{k}(t)\right)^{2}+\left(u-U_{k}(t)\right)^{2}}\right) .
$$

Then we want that all the integrals of the sums in 3.4 and 3.6 over each subdomain of the phase space to be almost equal. From the numerical point of view, we want to verify this proximity only for the support $C_{i}$ of functions $\gamma_{i}^{\alpha}$ that is

$$
\forall i \in\left\{1, \ldots, N_{p}\right\}, \quad \int_{C_{i}} \sum_{k=1}^{N_{p}} \widetilde{\beta}_{k}(t, \tau) \gamma_{k}^{\alpha}(q, u) d q d u=\int_{C_{i}} \sum_{k=1}^{N_{p}} \beta_{k}(t, \tau, q, u) \lambda_{k}^{\alpha}(q, u) d q d u
$$


where $\lambda_{k}^{\alpha}:(q, u) \mapsto\left(\gamma^{\alpha}\right)^{\prime}\left(\sqrt{\left(q-Q_{k}(t)\right)^{2}+\left(u-U_{k}(t)\right)^{2}}\right)$. System 3.7 writes again

$$
\forall i \in\left\{1, \ldots, N_{p}\right\}, \quad \sum_{k=1}^{N_{p}} \widetilde{\beta}_{k}(t, \tau) \int_{C_{i}} \gamma_{k}^{\alpha}(q, u) d q d u=\int_{C_{i}} \sum_{k=1}^{N_{p}} \beta_{k}(t, \tau, q, u) \gamma_{k}^{\alpha}(q, u) d q d u
$$

Therefore we can determine the coefficient $\widetilde{\beta}_{k}$ of the particle approximation of $W$ up to the solution of the linear system 3.8 .

Finally, we consider the last step of the algorithm. We want to build a particle approximation of $G_{1}$ from problem (2.9). We first note that in this problem, the advection operator is exactly the same as in problem (2.6). Therefore the macroparticles to approximate $G_{1}$ can be chosen as being localized exactly at the same phase space locations as the ones used to approximate $G$. Thus $G_{1}$ is approximated by

$$
G_{1}(t, q, u)=\sum_{k=1}^{N_{p}} \omega_{k}^{1}(t) \delta\left(q-Q_{k}(t)\right) \delta\left(u-U_{k}(t)\right),
$$

where the weights $\omega_{k}^{1}$ now depend on time $t$, which allows to take into account the effect of the source term on the right-hand side of the equation in 2.9 . Once more, we can regularize this approximation by

$$
G_{1}(t, q, u)=\sum_{k=1}^{N_{p}} \omega_{k}^{1}(t) \gamma^{\alpha}\left(\sqrt{\left(q-Q_{k}(t)\right)^{2}+\left(u-U_{k}(t)\right)^{2}}\right) .
$$

Then we handle the right-hand side of equation in problem 2.9 exactly as for $W$. Indeed, the approximation of this term has the following shape

$$
\sum_{k=1}^{N_{p}} \eta_{k}(t, q, u)\left(\gamma^{\alpha}\right)^{\prime}\left(\sqrt{\left(q-Q_{k}(t)\right)^{2}+\left(u-U_{k}(t)\right)^{2}}\right) .
$$

Therefore in order to distribute this approximation on the macroparticles, we want to determine $\widetilde{\eta}_{k}$ such that

$$
\forall i \in\left\{1, \ldots, N_{p}\right\}, \quad \int_{C_{i}} \sum_{k=1}^{N_{p}} \widetilde{\eta}_{k}(t) \gamma_{k}^{\alpha}(q, u) d q d u=\int_{C_{i}} \sum_{k=1}^{N_{p}} \eta_{k}(t, q, u) \lambda_{k}^{\alpha}(q, u) d q d u,
$$

which writes again

$$
\forall i \in\left\{1, \ldots, N_{p}\right\}, \quad \sum_{k=1}^{N_{p}} \widetilde{\eta}_{k}(t) \int_{C_{i}} \gamma_{k}^{\alpha}(q, u) d q d u=\int_{C_{i}} \sum_{k=1}^{N_{p}} \eta_{k}(t, q, u) \lambda_{k}^{\alpha}(q, u) d q d u .
$$

Then we can determine the coefficient $\widetilde{\eta}_{k}$ up to the solution of the linear system 3.13 . Since $G_{1}$ is solution to problem $(2.9)$, we finally have that

$$
\forall k \in\left\{1, \ldots, N_{p}\right\}, \quad \frac{d \omega_{k}^{1}}{d t}=\widetilde{\eta}_{k},
$$

that needs to be numerically solved to get the full approximation of $G_{1}$. 


\section{REFERENCES}

[1] G. Allaire. Homogenization and Two-scale Convergence. SIAM Journal on Mathematical Analysis, 23(6):14821518, 1992.

[2] E. Frénod, F. Salvarani, and E. Sonnendrücker. Long time simulation of a beam in a periodic focusing channel via a two-scale PIC-method. Mathematical Models and Methods in Applied Sciences, 19(2):175-197, 2009.

[3] E. Frénod and E. Sonnendrücker. The Finite Larmor Radius Approximation. SIAM J. Math. Anal., 32(6):1227$1247,2001$.

[4] A. Mouton. Two-scale semi-lagrangian simulation of a charged particles beam in a periodic focusing channel. Kinet. Relat. Models, 2(2):251-274, 2009.

[5] G. Nguetseng. A general convergence result for a functional related to the theory of homogenization. SIAM Journal on Mathematical Analysis, 20(3):608-623, 1989.

[6] G. Nguetseng. Asymptotic analysis for a stiff variational problem arising in mechanics. SIAM Journal on Mathematical Analysis, 21(6):1394-1414, 1990. 